\title{
Differential effects of GLI2 and GLI3 in regulating cervical cancer malignancy in vitro and in vivo
}

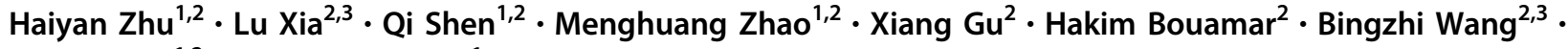 \\ Lu-Zhe Sun ${ }^{1,2} \cdot$ Xueqiong Zhu $\mathbb{1}^{1}$
}

Received: 21 September 2017 / Revised: 7 May 2018 / Accepted: 9 May 2018 / Published online: 2 July 2018

(c) United States \& Canadian Academy of Pathology 2018

\begin{abstract}
Advanced, recurrent, or persistent cervical cancer is often incurable. Therefore, in-depth insights into the molecular mechanisms are needed for the development of novel therapeutic targets and the improvement of current therapeutic strategies. In this study, we investigated the role of GLI2 and GLI3 in the regulation of the malignant properties of cervical cancer. We showed that down-regulation of GLI2, but not GLI3, with an inducible GLI2 shRNA inhibited the growth and migration of cervical cancer cell lines, which could be rescued by ectopic expression of GLI2. GLI2 appeared to support cell growth by regulating the mitosis, but not the apoptosis, of the cervical cancer cells. Mechanistically, these functions of GLI2 were in part mediated by the activation of AKT pathway. Knockdown of GLI2, but not GLI3, also inhibited xenograft growth of cervical cancer cells in vivo. Finally, analysis of TCGA data showed that high levels of GLI2, but not GLI3, conferred a poor prognosis in cervical cancer patients. These observations for the first time suggest that GLI2, but not GLI3, exerts a tumor-promoting role in cervical cancer and may be targeted as a novel therapeutic strategy.
\end{abstract}

\section{Introduction}

Cervical cancer remains a major health problem worldwide [1]. It is the second most commonly diagnosed cancer and third leading cause of cancer death among females in developing countries. Even in the United States with the widespread undertake of screening for the prevention and

These authors contributed equally: Haiyan Zhu, Lu Xia

Electronic supplementary material The online version of this article (https://doi.org/10.1038/s41374-018-0089-5) contains supplementary material, which is available to authorized users.

\section{Lu-Zhe Sun}

sunl@uthscsa.edu

$\triangle$ Xueqiong Zhu

zjwzzxq@163.com

1 Department of Obstetrics and Gynecology, the Second Affiliated Hospital of Wenzhou Medical University, Wenzhou, China

2 Departments of Cell Systems \& Anatomy, School of Medicine, University of Texas Health Science Center, San Antonio, TX, USA

3 Xiangya Hospital and Xiangya School of Medicine, Central South University, Hunan, China early detection of cervical cancer, $\sim 12,900$ new cases and 4100 deaths from cervical cancer were estimated for 2015 [1]. Though early-stage cervical cancer is typically amenable to cure, the diagnosis of advanced, recurrent, or persistent cervical cancer is often incurable. Therefore, the indepth insights into the molecular mechanisms that contribute to its progression are needed for the development of novel therapeutic targets and the improvement of current strategies to cure cervical cancers.

The Hedgehog (Hh) pathway is an evolutionarily conserved pathway responsible for many aspects of embryonic development and stem cell maintenance. Recently, this pathway is found to be aberrantly activated in a spectrum of malignancies [2]. In the absence of the Hh ligands, the Patched 1 protein (PTCH1) represses the activity of smoothened (SMO), thus silencing intracellular signaling. On ligand binding, PTCH1 relieves the inhibition of SMO resulting in SMO activation [3-5]. Activated SMO stimulates a singling cascade that eventually results in activation and translocation of the GLI transcription factors into nucleus, where they activate the expression of $\mathrm{Hh}$ target genes [3-5].

There are three members in the GLI transcription factor family in vertebrates, i.e. GLI1, GLI2, and GLI3 [6]. These three GLI transcription factors are likely derived from 
duplications of a single ancestral GLI gene [6]. All GLI proteins contain a C-terminal activator domain, whereas GLI2 and GLI3 possess an N-terminal repressor domain [4]. Thus, while GLI1 exclusively acts as a transcription activator, GLI2 and GLI3 can act either as repressors or activators. GLI family has been reported to be overexpressed in cervical cancer [7-9]. The expression of GLI1 correlated with the late clinical stage, poor differentiation and lymph node metastasis [8] and co-inhibition of GLI1 and E6 in cervical cancer cells showed additive anti-cancer effects [10]. In contrast to GLI1, there is less data to implicate GLI2 and GLI3, which act either as repressors or activators, in cervical cancer. Therefore, in this study, we investigated the role of GLI2 and GLI3 in the regulation of the malignant properties of cervical cancer and whether knockdown of GLI2/3 can suppress cervical cancer growth both in vitro and in vivo. We demonstrated that downregulation of GLI2, but not GLI3, inhibited cervical cancer growth both in vitro and in vivo. Mechanistically, these functions were in part mediated by the activation of AKT. Moreover, analysis of TCGA data revealed that high levels of GLI2, but not GLI3, conferred a poor prognosis in cervical cancer patients. These observations suggest that GLI2, but not GLI3, exerts a tumor-promoting role in cervical cancer.

\section{Materials and methods}

\section{Cell cultures}

Human cervical cancer cell lines, HeLa, Caski, and C-4I, were originally obtained from the American Type Culture Collection (ATCC). All three cell lines were authenticated with the DNA markers used by ATCC. The cultures were maintained in RPMI 1640 medium (Invitrogen, Carlsbad, CA, USA) supplemented with $10 \%$ fetal bovine serum (FBS) and $0.1 \%$ penicillin in $5 \% \mathrm{CO}_{2}$ at $37^{\circ} \mathrm{C}$.

\section{Construction of the Tet inducible GLI2/3-specific short hairpin RNA expression plasmid}

The lentiviral vector Tet-pLKO-puro (Addgene plasmid 21915) was used to generate inducible GLI2 or GLI3targeting shRNA constructs. In brief, DNA oligos targeting human GLI2 (5'CCGGCCTGGCATGACTACCACTATG CTCGAGCATAGTGGTAGTCATGCCAGGTTTTTG 3') or GLI3 (5'CCGGCGGAAATCAATAGGAGTTGAACTC GAGTTCAACTCCTATTGATTTCCG TTTTT3') were designed and cloned into the Tet-pLKO-puro cloning vector digested with AgeI and EcoRI. The recombinant plasmid was named pTet-GLI2shR (GLI2 shR) or pTet-GLI3shR (GLI3 shR2). To construct a control vector, Tet-pLKO-puro vector was digested with AgeI and EcoRI to release the stuffer segment, following which $5^{\prime}$ overhang ends were blunted with Klenow DNA polymerase (New England Biolabs) and the segment was then ligated using T4 ligase (New England Biolabs). This vector was named pTet-ctl (Ctl shR1). The recombinant construct was co-transfected together with two packaging vectors psPAX2 and pMD2.G into $293 \mathrm{~T}$ cells. Lentiviral particles were harvested and filtered to infect cervical cancer cell lines followed with puromycin selection for stably infected cells.

\section{GLI2/3-specific short hairpin RNA expression plasmid}

Plasmids pLKO.1-GLI3 (GLI3 shR1), pLKO.1-GLI2 (GLI2 shR2), and the matched vector pLKO.1 (Ctl shR2) were purchased from Sigma (St. Louis, MO). These vectors were co-transfected together with two packaging vectors psPAX2 and pMD2.G into 293T cells. Lentiviral particles were harvested and filtered to infect cervical cancer cell lines followed by puromycin selection. The sequence of GLI3 shR1 as following: CCGGACAAGAGGTCCA AGATCAAACCTCGAGGTTTGATCTTGGACCTCT TG TTTTTTTG. The sequence of GLI2 shR2 as following: CCGGCTGGACAGGGATGACTGTAAGCTCGAGCT TACAGTCATCCCTGTCCAGTTTTTG.

\section{Construction of $\Delta \mathrm{N}-\mathrm{GLI} 2$ expression and Myr-AKT plasmid}

$\triangle \mathrm{N}-\mathrm{GLI} 2 \mathrm{cDNA}$ or myr-AKT cDNA were subcloned into the Pmel site of the pSDM101-green fluorescent protein (GFP) vector. $\triangle \mathrm{N}$-GLI2-pSDM-GFP, myr-AKT-pSDMGFP (AKT cDNA), and Psdm101-GFP (Ctl vector) lentiviral supernatants were produced in 293T cells as described above. $\Delta \mathrm{N}-\mathrm{GLI} 2$ is the full length GLI2 lacking 328 amino acid at its $\mathrm{N}$ terminus that has repressor activity [11]. It is more transcriptionally active than full length GLI2 without the repressor domain.

\section{Western immunoblotting analysis}

Both cell and tissue samples were homogenized and lysed in Laemmli buffer with a cocktail of protease inhibitors. The total protein concentrations were quantified by the bicinchoninic acid protein assay (Thermo Scientific, Rockford, IL). Equal amounts of total protein were resolved by sodium dodecyl sulfate-polyacrylamide gel electrophoresis, transferred to a nitrocellulose membrane under constant voltage and blocked with Tris buffered saline with Tween (TBST) containing 5\% non-fat dried milk. Primary antibodies and secondary antibodies were diluted in TBST or $3 \%$ non-fat dried milk and applied with a washing step in 
between. Proteins were detected using the Amersham ECL. Western blotting detection kit (GE Healthcare, Piscataway, NJ). Primary antibodies used including: GLI2 (Novus Biologicals, Littleton, CO), GLI3 (Fisher Scientific, Waltham, MA, both the full length (GLI3FL) and repressor (GLI3R) versions of GLI3 protein can be detected by this antibody), p21 (Santa Cruz Biotechnology, Santa Cruz, CA), p27 (Santa Cruz Biotechnology, Santa Cruz, CA), Cyclin D1 (Santa Cruz Biotechnology, Santa Cruz, CA), pAKT (Ser473, Cell signaling, Danvers, MA), total-AKT (Cell signaling, Danvers, MA), GAPDH and Tublin (Santa Cruz Biotechnology, Santa Cruz, CA).

\section{RNA extraction and quantitative real-time PCR}

The mRNA levels were measured by quantitative real-time PCR. RNA extracted from the cultured cells or xenograft tumors was treated with DNase1 (Invitrogen, Grand Island, $\mathrm{NY}$ ) to remove genomic DNA contamination. Total RNA $(2 \mu \mathrm{g})$ was reverse-transcribed into cDNA using random primers and M-MLV reverse transcriptase from Invitrogen Life Technology (Grand Island, NY). Quantitative real-time PCR (qRT-PCR) was performed using Power SYBR Green PCR Mix from Life Technologies. All primers used in this study were designed by Primer Blast of NCBI and synthesized by Integrated DNA Technologies (Coralville, IA). Primer pair specificity was determined by generation of a single peak for dissociation curve (melting curve) at the end of real-time PCR cycling program. GAPDH was used as the internal control. The primers used in this study are as follows: GLI1-forward primer, 5'-CAT CAACAGCGAGCA CATCC-3', GLI1-reverse primer, 5'-ACATACATACGGC TTCTCATTGG-3'; GLI2- forward primer, 5'-GGGTCAA CCAGGTGTCCAGCACTGT-3', GLI2-reverse primer, GATGGAGGGCAGGGTCAAGGAGTTT; GLI3-forward primer, 5'-TGGTCGAAGAGAGCTGAA GTA-3'; GLI 3-reverse primer, 5'-CTGTGGCTGCATAGTGATTGC-3'.

\section{Cell proliferation assay}

Cells were plated in a 96-well plate at 2000 cells/well with or without $1 \mu \mathrm{M}$ doxycycline in triplicates. After every consecutive day for 5-7 days, 3-(4,5-dimethylthiazol-2-yl)2,5-diphenylte-trazolium bromide solution (MTT, $2 \mathrm{mg} / \mathrm{ml}$ in PBS) was added and the plates were incubated at $37{ }^{\circ} \mathrm{C}$ for $2 \mathrm{~h}$ in a tissue incubator. The blue colored formazan product was dissolved in dimethyl sulfoxide (DMSO) and measured at $595 \mathrm{~nm}$ wavelength on a Biotek plate reader.

\section{Soft-agar colony formation assay}

Cells in $250 \mu \mathrm{l} 0.4 \%$ soft agarose (Life Technologies, Carlsbad) mixed with $10 \%$ FBS-containing medium were plated at 2000/well on top of a $500 \mu \mathrm{l}$ solidified underlayer of $0.8 \%$ agarose in the same medium in a 24 -well tissue culture plate. Doxycycline $(1 \mu \mathrm{M})$ or equivalent control vehicle, DMSO, were administrated after 5 days when small colonies formed. After 2-3 weeks of incubation, cell colonies were visualized by overnight staining with 1 $\mathrm{ml} p$-iodonitrotetrazolium violet staining (Sigma, St. Louis, MO, USA). Then, we scanned stained soft agarose plates with a scanner and colony number was quantified using Image J.

\section{Cell migration assay}

Cell migration assays were performed in 24-well transwells with $8-\mu \mathrm{m}$ pore polycarbonate membranes (BD Biosciences, San Diego, CA). Cells at a density of 20,000-40,000 cells/well in serum-free medium were seeded in the upper insert in triplicates. The lower chamber contained the medium with $10 \%$ of FBS as chemoattractant. After incubation with $5 \% \mathrm{CO}_{2}$ at $37^{\circ} \mathrm{C}$ for $18 \mathrm{~h}$ (Caski, C4Icell) or $6 \mathrm{~h}$ (HeLa cell), the cells that did not migrate across the membrane were removed with a cotton swab and the migrated cells were stained with the Hema 3 Stain 18 kit (Fisher Scientific, Waltham, MA) according to the manufacturer's protocol. Migrated cells were counted under a microscope with $100 \times$ magnification.

\section{Cell cycle analysis}

Cells were plated at $5 \times 10^{5}$ cells/dish in $60 \mathrm{~mm}$ dishes with or without $1 \mu \mathrm{M}$ doxocycline treatment for $48 \mathrm{~h}$, followed by collection by trypsinization. The cells then were fixed with $100 \%$ ethanol, stained with $10 \mu \mathrm{g} / \mathrm{ml}$ propidium iodide in the presence of $5 \mu \mathrm{g} / \mathrm{ml}$ RNase A and analyzed for fluorescence using a FACScan flowcytometer (Becton Dickinson, San Diego, CA).

\section{ELISA}

Cells were plated at $5 \times 10^{5}$ cells $/$ dish in $60 \mathrm{~mm}$ dishes and harvested after various treatments. Cell pellets were washed with cold $1 \times$ PBS twice, lysed with $40 \mu \mathrm{l}$ apoptosis lysis buffer and apoptosis was assayed using an apoptosis detection kit (Roche Applied Science, Indianapolis, IN), which is based on the measurement of histone-complexed DNA fragments in the cytoplasm of apoptotic cells, according to the manufacture's protocol.

\section{Annexin-V FITC staining}

Cells were plated at $5 \times 10^{5}$ cells/dishes into $60 \mathrm{~mm}$ dishes. After reaching $70-80 \%$ confluence during exponential growth, cells were harvested, washed with cold PBS and 
resuspended with binding buffer at a concentration of $2 \times$ $10^{6} \mathrm{cell} / \mathrm{ml}$. Cells were analyzed by using the ApoTarget ${ }^{\mathrm{TM}}$ Annexin-V FITC Apoptosis kit (Invitrogen, Grand Island, $\mathrm{NY}$ ) according to the manufacture's protocol.

\section{Immunohistochemistry}

Immunohistochemistry staining was performed on paraffinembedded $4 \mu \mathrm{m}$ tissue sections mounted on poly-L-lysinecoated slides. Briefly, after deparaffinization in xylenes and rehydration through graded ethanol solutions, antigen retrieval was performed by submerging the sections into a sodium citrate solution $(10 \mathrm{mM}, \mathrm{pH} 6.0)$ or a EDTA solution $(1 \mathrm{mM}, \mathrm{pH} 8.0)$ at $95^{\circ} \mathrm{C}$ for 15 or $30 \mathrm{~m}$, in a microwave oven. The tissue sections were then treated with $3 \%$ hydrogen peroxide in methanol to suppress the endogenous peroxidase activity. Tissue sections were then incubated with antibodies to GLI2 (Novus Biologicals, Littleton, CO), and Ki-67 (BD Pharmingen, San Diego, CA), at $4{ }^{\circ} \mathrm{C}$ overnight. After washing, the sections were incubated with pre-diluted secondary antibody (BD Pharmingen, San Diego, CA), followed by further incubation with 3,3-diaminobenzidine tetrahydrochloride. Finally, the slides were counterstained with hematoxylin and mounted in an aqueous mounting medium. Appropriate positive and negative controls were stained in parallel. For negative controls, primary antibodies were replaced with PBS.

\section{Animal studies}

Four-week-old female nude mice were used for in vivo animal experiments. The animals were housed under specific pathogen-free condition. Exponentially growing Caski GLI2 shR cells or Ctl shR1 cells $\left(1.6 \times 10^{6}\right.$ cells $/ 120 \mu \mathrm{l} /$ mouse) suspended in cold PBS were injected subcutaneously into the right flank of the mice. After tumor cell inoculation for 1-2 weeks, growing tumors were observed and their size was recorded twice a week. The length and width of each tumor were measured using a caliper, and the volumes were calculated by the following formula: volume $\left(\mathrm{mm}^{3}\right)=$ length $\times$ width $\times$ width $/ 2$. After another 2-3 weeks, mice with tumor burden $\geq 100 \mathrm{~mm}^{3}$ in volume were ranked and divided into two groups (10 mice for each group) with matched mean tumor volumes and treated as follows: $2.5 \%$ sucrose-containing drinking water with or without $1 \mathrm{mg} / \mathrm{ml}$ of doxycycline. Doxycycline was administered via drinking water. Water was replenished every 4 days. After treatment for 18 days (Ctl shR1) or 33 days (GLI2 shR), xenograft tumors were isolated from mice. A portion of the tumor tissue was fixed in $4 \%$ paraformaldehyde for histological study, and the rest was frozen for other experiments.

Similarly, exponentially growing Caski GLI3 shR1 cells or $\mathrm{Ctl}$ shR2 cells $\left(1.6 \times 10^{6}\right.$ cells $/ 120 \mu \mathrm{l} /$ mouse $)$ suspended in cold PBS were injected subcutaneously into the right flank of the mice. After tumor cell inoculation for 2 weeks, growing tumors were observed and their size was recorded twice a week. Forty-eight days later, all the mice were sacrificed. A portion of the tumor tissue was fixed in $4 \%$ paraformaldehyde for histological study, and the rest was frozen for other experiments.

\section{The Cancer Genome Atlas (TCGA) dataset}

The Cancer Genome Atlas (TCGA) database (https://gdcportal.nci.nih.gov/) was downloaded for RNA sequencing data, including 307 cervical cancer samples (there are no GLI2/3 expression data for three patients). After normalization, Limma and edgeR packages in $\mathrm{R}$ language were used to identify the genes differential expression. $\mathrm{R}$ language was used to get Kaplan-Meier survival plots to explore the association between individual GLIs mRNA levels and overall survival (OS) of cervical cancer patients. The downloaded TCGA data is shown in supplementary Table 1 and our R code is in supplementary Table 2 .

\section{Statistical analyses}

Two-tailed Student's $t$-test was used to compare the means of two groups. One-way ANOVA analysis of variance with Tukey-Kramer post hoc test was used for analyzing data when means from more than two groups were compared. Results are expressed as mean \pm SEM. $P<0.05$ was considered to be statistically significant. All the statistical analyses were performed with Graph Pad Prism 3.03 software.

\section{Results}

\section{GLI transcription factors are expressed in cervical cancer}

In order to determine the role of GLI family in the cervical carcinogenesis, we first examined mRNA levels of the three GLI transcription factors, GLI1, GLI2, and GLI3, in three human cervical cancer lines, by using qRT-PCR. As shown in Fig. 1, certain mRNA levels of GLI1, GLI2, and GLI3 were observed in all three cervical cancer cell lines. It was remarkable that the expression of GLI2 and GLI3 was much higher than GLI1. We further analyzed the mRNA levels of GLI transcription factors in 304 human cervical cancer tissues using TCGA database. Similarly, we observed that mRNA levels of GLI2 and GLI3 was significantly higher than GLI1 (Fig. 1d). Clearly, these results indicated that GLI transcription factors were expressed in cervical cancer and GLI2/3 mRNA levels were much higher than GLI1. 
Fig. 1 GLI1, GLI2, and GLI3 were expressed in cervical cancer cell lines and cervical cancer tissues. Transcript levels of (a) GLI1, (b) GLI2, and (c) GLI3 were illustrated for the three cervical cancer cell lines, HeLa, Caski, and C-4I. Data presented were mean \pm SEM of three replicate measurements. d Plot of $\log _{2}$ transformed and mean \pm SEM centered GLI1, GLI2, and GLI3 mRNA levels in 304 human cervical cancer tissues using TCGA database. Data presented were mean \pm SEM. $* * * P<0.001$ with oneway ANOVA and

Tukey-Kramer post hoc test
A

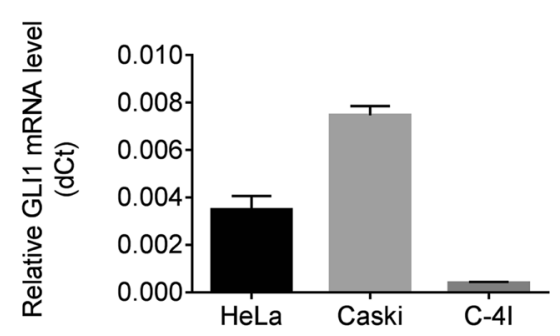

C

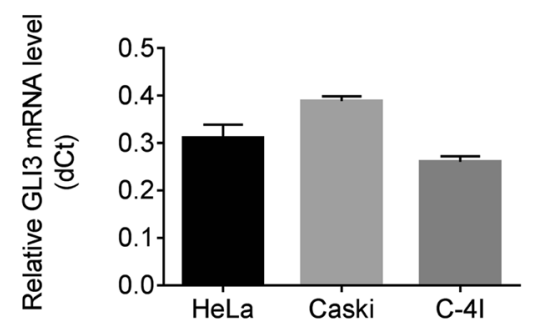

B

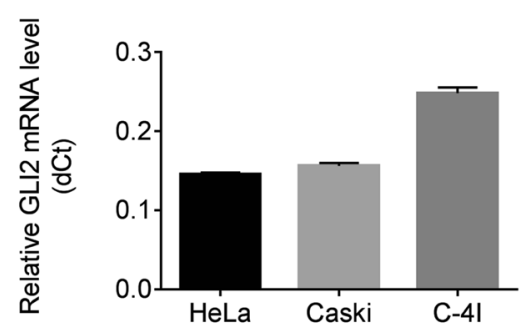

D
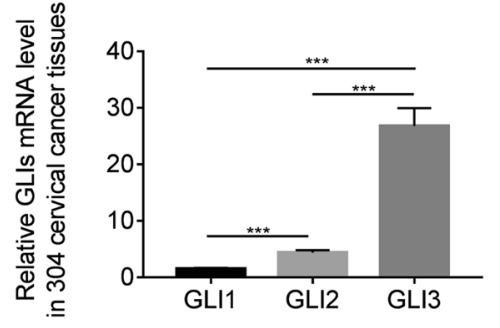

\section{Knockdown of GLI2 inhibits cell proliferation and migration in vitro}

To determine whether GLI2 and GLI3 promoted cervical cancer progression, we first used a doxycycline inducible shRNA targeting GLI2 (GLI2 shR) and a matched control shRNA (Ctl shR1) to knockdown GLI2 expression in various cervical cancer cell lines. Reduced expressions of GLI2 was confirmed by qRT-PCR in mRNA level and by western blotting in protein level after the cells were treated with doxycycline (Fig. 2a, b). MTT assays revealed that depletion of GLI2 significantly inhibited the growth of cervical cancer HeLa and Caski cells on day 5 and 7 while the effect on C-4I cell growth was less dramatic (Fig. 2c). The malignancy-promoting role of GLI2 was further demonstrated with soft agar colony formation assay in HeLa cell line (Fig. 2d). Additionally, GLI2 knockdown also significantly inhibited migration in all of the three cervical cancer cell lines (Fig. 2e). To verify these results, we also used a second GLI2 shRNA (GLI2 shR2) lenti vector, which was stably transduced in the HeLa cell line and confirmed that knockdown of GLI2 inhibited cell proliferation on plastic and in soft agar, as well as migration of the cervical cancer cells (Supplementary Figure 1).

\section{Re-expression of GLI2 rescues the GLI2 knockdown- associated phenotypes}

We next investigated whether ectopic expression of GLI2 by lentiviral delivery could promote the malignant phenotypes and rescue the effect of GLI2 knockdown by shRNA. We first transfected the GLI2-shRNA-HeLa and GLI2shRNA-Caski cell lines with GLI2 cDNA or a matched control vector (Ctl vector), and then treated the cells with or without doxycycline for $48 \mathrm{~h}$ to induce the knockdown of GLI2. Over-expression and knockdown of the GLI2 protein and mRNA were confirmed by western blotting analysis and qRT-PCR respectively (Fig. 3a, b). As expected, overexpression of GLI2 in HeLa and Caski cells promoted the proliferation by MTT assays and migration by transwell assays (Fig. 3c, d). Importantly, GLI2 expression partly rescued the effects of GLI2 knockdown by shRNA, indicating the specificity of the shRNA approach and pointing to GLI2 as a key regulator of cell proliferation and migration in cervical cancer cells.

\section{Knockdown of GLI2 induces cell-cycle arrest at G0/ G1 phase}

The above results demonstrated GLI2 expression might increase the proliferative capacity of cervical cancer cells. To extend these findings, we performed cell cycle analysis by flow cytometry and apoptosis analysis by ELISA assay and Annexin-V FITC staining. Flow cytometry analysis of cell-cycle distribution revealed that depletion of GLI2 led to a G0/G1 arrest, with an increase in the percentage of cells in the G0/G1 phase of the cell cycle and a concomitant decrease in the number of cells in the $\mathrm{S}$ phase (Fig. 4a). Furthermore, silencing GLI2 increased the expression of p21 and p27, and decreased the expression of cyclin D1 (Fig. 4b). Interestingly, depletion of GLI2 did not affect apoptosis by ELISA assay (Fig. 4c) and Annexin-V FITC staining (Fig. 4d). Thus, silencing GLI2 regulated cervical cancer cell growth by inducing G0/G1 cell cycle arrest, but not inhibiting apoptosis. 

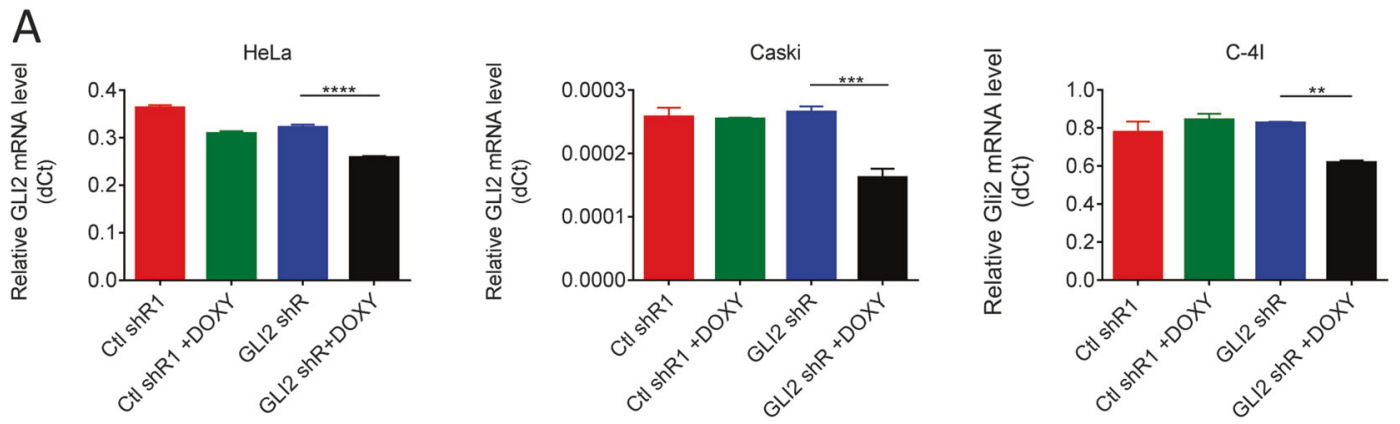

B

HeLa

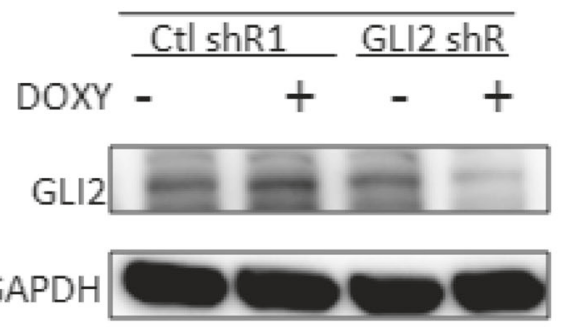

Caski

C $\rightarrow$ Ctl shR1 HeLa

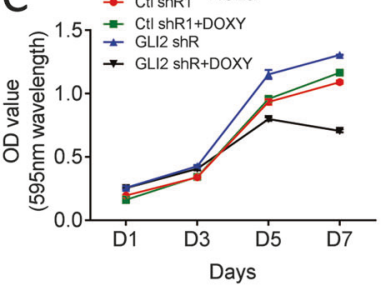

D

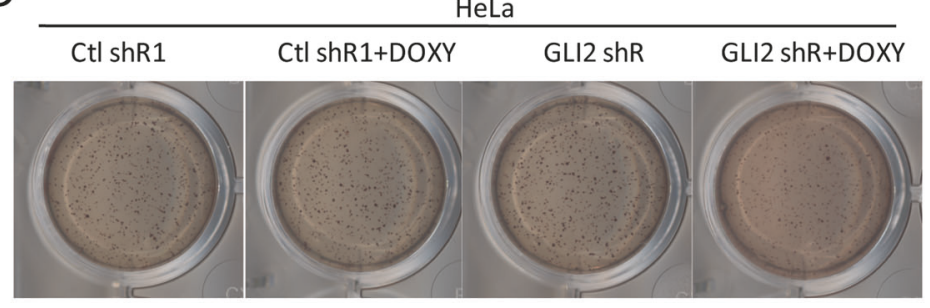

$E$

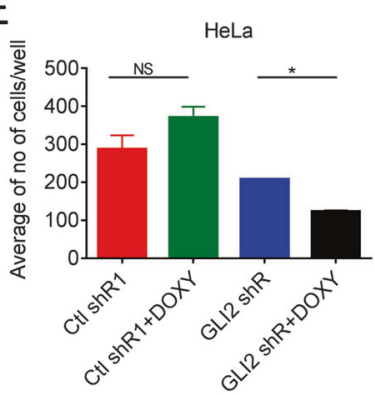

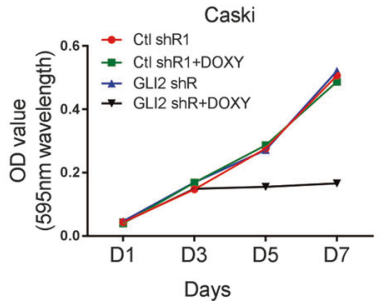

HeLa

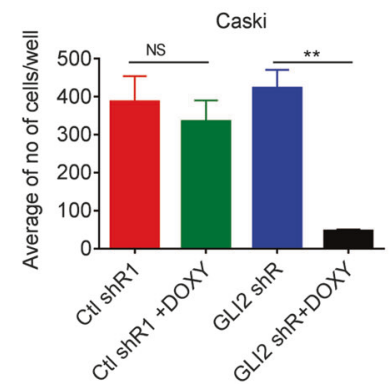

C-4I

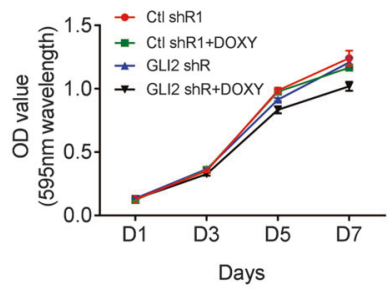

HeLa
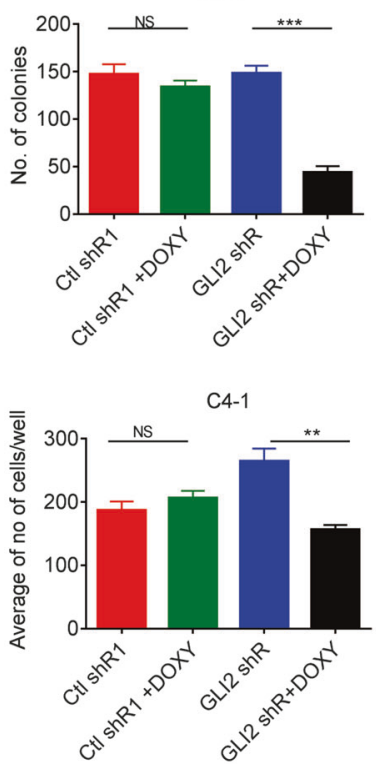

Fig. 2 Knockdown of GLI2 inhibited proliferation and migration in vitro. Inducible GLI2 shRNA and matched control shRNA were transfected into HeLa, Caski, and C-4I cells via lentiviral infection. a Confirmation of GLI2 stable knockdown in cervical cancer cell lines in its transcription level with qRT-PCR. GAPDH transcript was used for normalization. b GLI2 knockdown was confirmed in its protein level with Western blotting. GAPDH protein level was used to validate equal sample loading. GLI2 knockdown inhibited cervical cell growth in MTT assay (c), anchorage-independent growth in soft agar assay (d), and migration in transwell assay (e). Data presented were mean \pm SEM from triplicate measurements. $* P<0.05$; $* * P<0.01$; $* * * P<$ 0.001 with one-way ANOVA and Tukey-Kramer post hoc test 
Fig. 3 Restoration of the phenotypes by re-expression of GLI2 in HeLa and Caski cells. Protein (a) and mRNA (b) levels of GLI2 in GLI2 shR-expressing HeLa and Caski cells transfected with a control vector (Ctl vector) or a GLI2 cDNA expression vector in the presence or absence of doxycycline. Tublin protein level was used to validate equal sample loading in the western blotting, whereas GAPDH transcript was used for normalization in the qRT-PCR assay. c MTT assay was performed to obtain OD values reflecting relative cell numbers of GLI2 knockdown and overexpressing cells described above. d Migration of GLI2 knockdown and overexpressing cells described above was measured after seeding for $6 \mathrm{~h}$ (HeLa) or $16 \mathrm{~h}$ (Caski) in Transwell assay. Data presented were mean \pm SEM from triplicate measurements. $* P<$ $0.05 ; * * P<0.01 ; * * * P<0.001$ with one-way ANOVA and Tukey-Kramer post hoc test
A

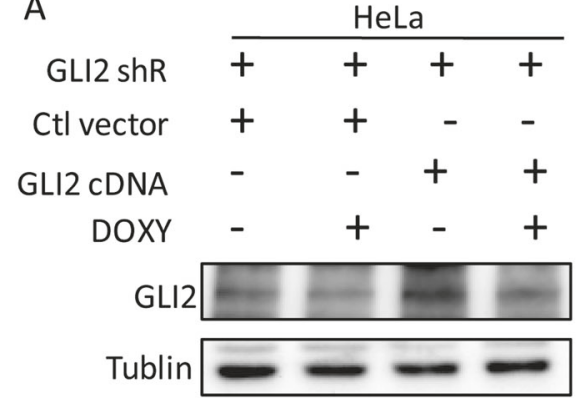

B

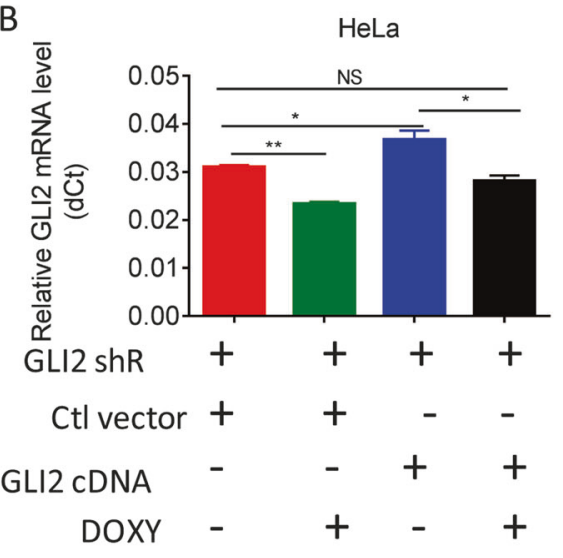

C

HeLa

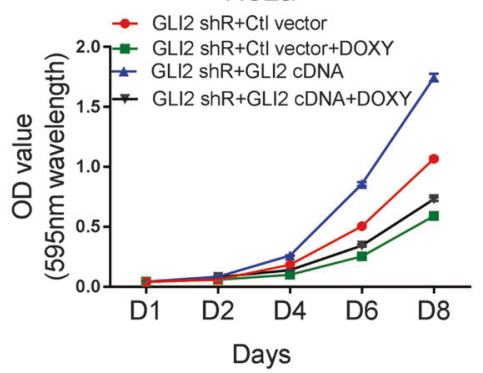

D

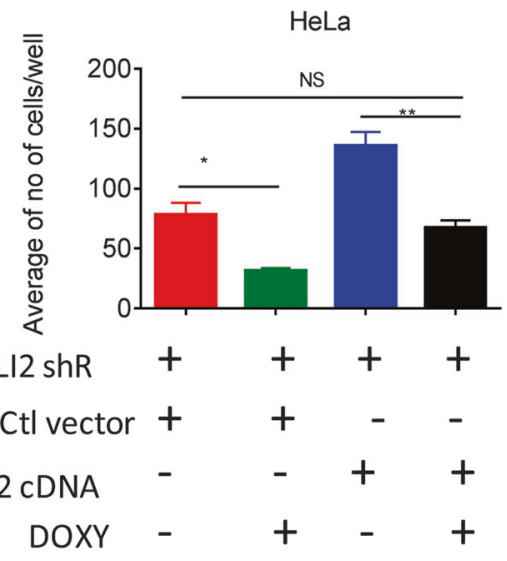

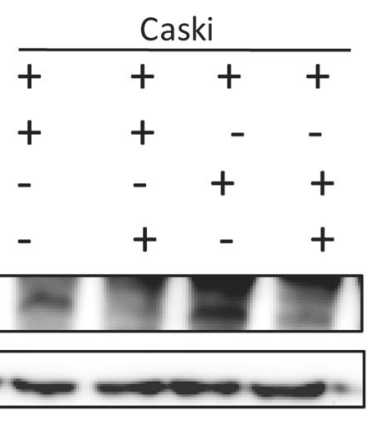

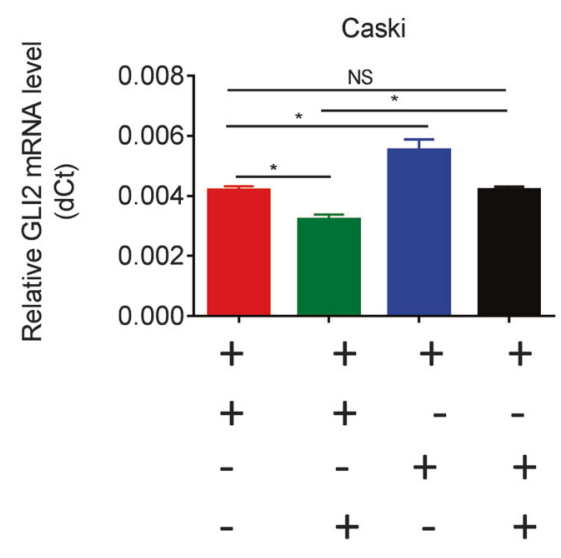

Caski

$\rightarrow$ GLI2 shR+Ctl vector
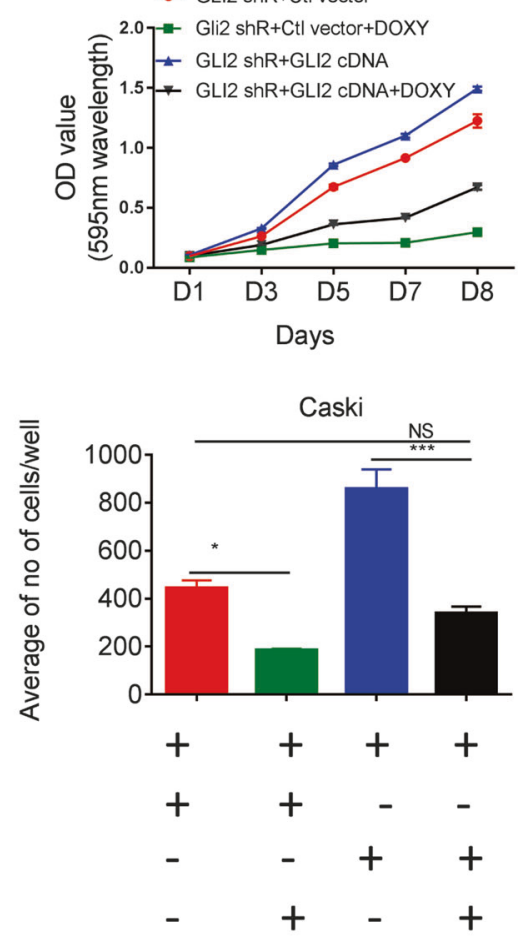
Fig. 4 Knockdown of GLI2 induced cell-cycle arrest at G0/ G1 with no effect on apoptosis. a Knockdown GLI2-induced cell cycle arrest at G0/G1 in caski cells. b Knockdown of GLI2 increased the expression of p21 and p27, and decreased the expression of cyclin D1 in Caski cells. GAPDH was used to validate equal sample loading. c, d Knockdown GLI2 had no effect on cells apoptosis by ELISA assay (c) and Annexin-V FITC staining (d). Data presented were mean \pm SEM from triplicate measurements. $* * * P<0.001$ with one-way ANOVA and Tukey-Kramer post hoc test
A
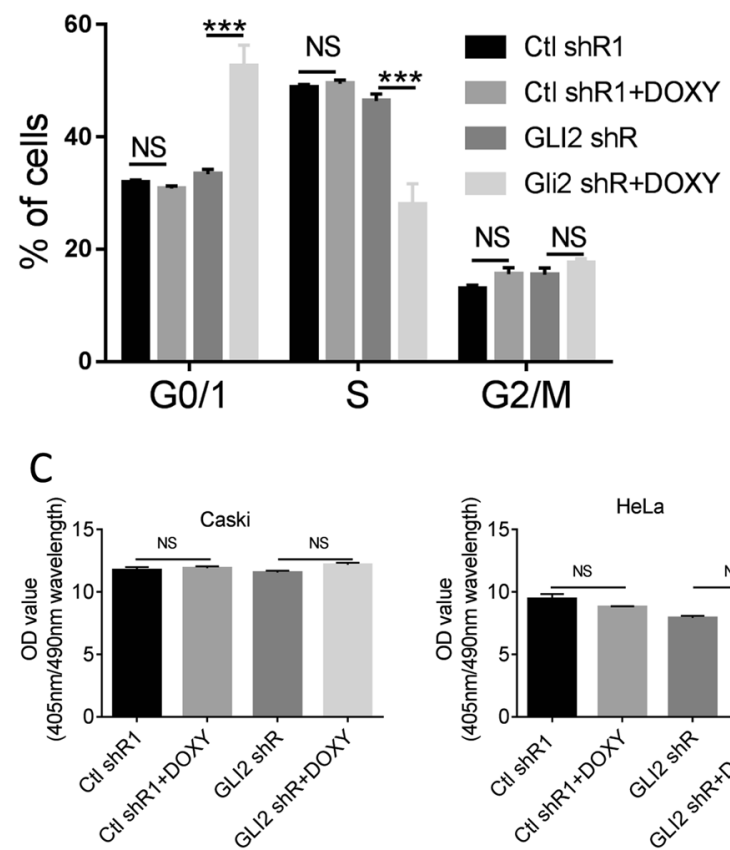

B

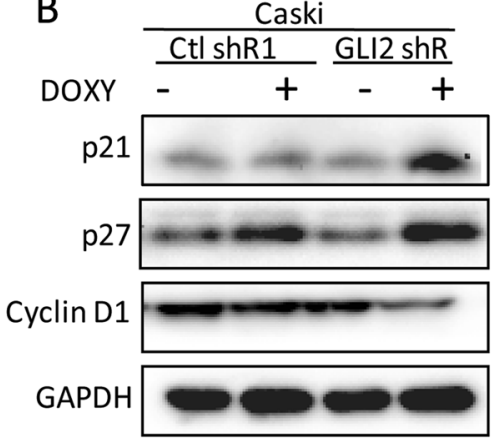

\section{Knockdown of GLI2 suppresses cell growth via inhibition of AKT activation}

\begin{abstract}
Aberrant over-activation of AKT via phosphorylation at Thr 308 is a common feature in both early and advanced cervical cancers [12-15]. To elucidate potential mechanistic pathways responsible for the malignant phenotypes induced by GLI2, we examined p-AKT status in Caski cell. While the total AKT level was basically the same in the GLI2 shRNA and Ctl shRNA Caski cell, its phosphorylation was noticeably reduced in GLI2 knockdown Caski cells (Fig. 5a). To further study whether AKT activation was necessary for GLI2-mediated promotion of cervical cancer cell growth, we stable transfected GLI2-shRNA-Caski cells with myr-AKT expression vector or a matched control vector (Ctl vector), and then treated with or without doxycycline (Fig. 5b). As shown in Fig. 5c, we noticed that myr-AKT-cDNA expression stimulated cell growth. Furthermore, co-transfection with GLI2 shRNA and myr-AKTcDNA rescued the phenotype due to GLI2 deprivation. Thus, the data was consistent with the conclusion that GLI2 promotes cell growth via activation of AKT.
\end{abstract}

\section{Stable knockdown of GLI3 has no effect on proliferation and migration}

To examine the role of GLI3 on proliferation and migration, HeLa, Caski, and C-4I were transfected with a shRNA targeting GLI3 (GLI3 shR1) and a matched control shRAN
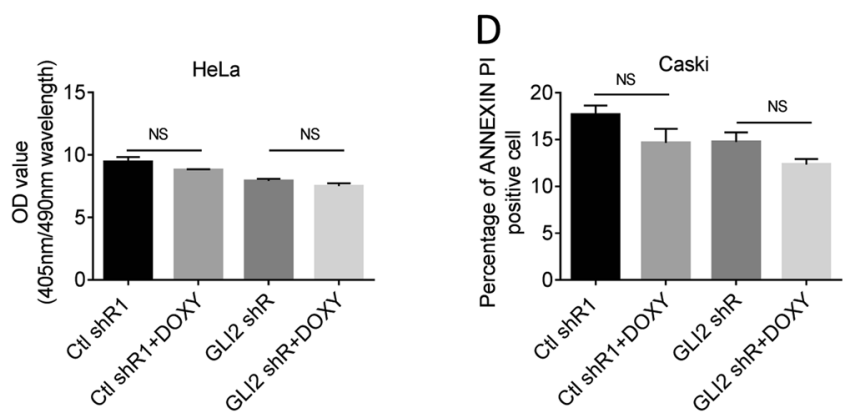

(Ctl shR2) (Fig. 6a, b). Intriguingly, knockdown of GLI3 had no significant effect on cell growth and migration of all the three cervical cancer cells tested (Fig.6c, d). To verify these results, we also used a Tet-inducible GLI3 shRNA (GLI3 shR2) stably transduced in Caski and C-4I cell lines and confirmed that knockdown of GLI3 did not affect the growth and migration of the cervical cancer cells (Supplementary Figure 2). These data suggested that GLI2 was specifically required for proliferation and migration in cervical cancer whereas GLI3 appeared dispensable.

\section{Knockdown of GLI2, but not GLI3, inhibits tumor growth in vivo}

To further substantiate the tumorigenic role of GLI2 in cervical cancer. We next developed a cervical cancer xenograft animal model by subcutaneous inoculation of shRNA-mediated GLI2 stable silencing Caski cells and a matched control shRNA Caski cells in female nude mice. Mice bearing growing GLI2-shR-Caski or Ctl-shR1-Caski xenografts were divided into two groups with similar mean tumor volumes of greater than $100 \mathrm{~mm}^{3}$ and then treated with or without doxycycline. As shown in Fig. 7a, b, while Caski Ctl shR1 (Ctl-shR1) treatment with or without doxycycline showed no effect on tumor growth, the tumor volumes of the Caski GLI2 shR treated with doxycycline were significantly lower than that of Caski GLI2 shR without doxycycline and the tumor volumes at the last two measurements were statistically different (Fig.7a). Tumor 


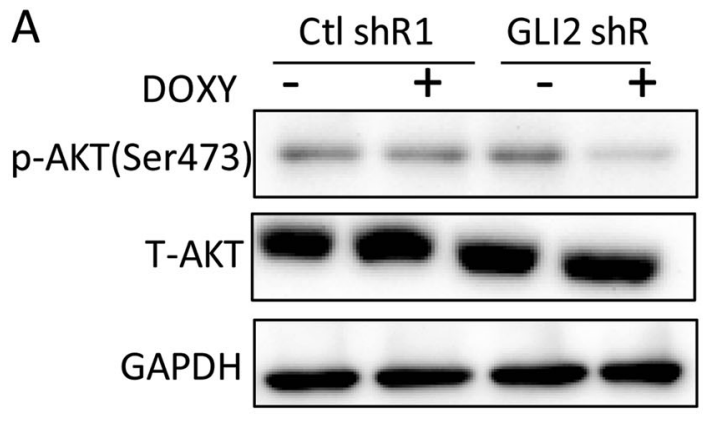

B
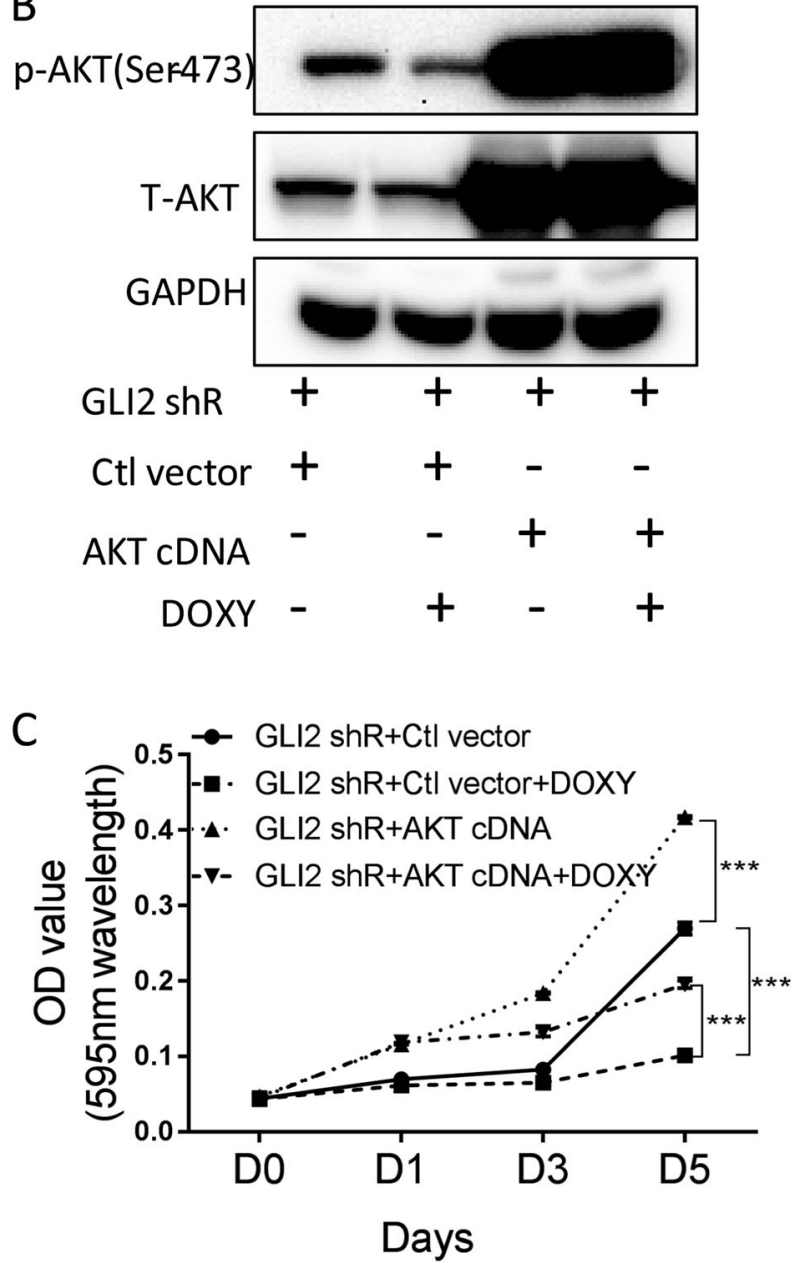

Fig. 5 Knockdown of GLI2 suppressed cell growth via inhibition of AKT. a Protein levels of phosphorylated (p-AKT, Ser473) and total (T-AKT) AKT in GLI2 knockdown and the matched control Caski cells. GAPDH level was used to validate equal sample loading. b Protein levels of p-AKT (Ser473) and T-AKT in GLI2 shRtransfected Caski cells without or with overexpression of myrAKTcDNA in the presence or absence of doxycycline. GAPDH level was used to validate equal sample loading. c MTT assay was performed to obtain OD values reflecting relative cell numbers of GLI2 transfected cells described above. Data presented were mean \pm SEM from triplicate measurements. $* * * P<0.001$ with two-way ANOVA and Tukey-Kramer post hoc test weight was also recorded when mice were euthanized. Consistent with final tumor volume, tumor weight of Caski GLI2 shR with doxycycline group was significantly reduced compared with Caski GLI2 shR without doxycycline group (Fig. 7b). Consistent with the inhibition of cell cycle progression in vitro (Fig. 4a) and tumor growth in vivo (Fig. 7a, b), GLI2 knockdown also reduced the number of cells undergoing proliferation, which showed positive $\mathrm{Ki}-67$ immunohistological (IHC) staining (Fig. 7c). We also confirmed the sustained knockdown of GLI2 in the tumors formed by Caski GLI2 shR cells treated with doxycycline. The number of nuclear GLI2-positive cells with IHC staining were significantly lower in doxycycline-treated Caski GLI2 shR cell-formed tumors than those in other cells-formed tumors (Fig. 7c). Taken together, these findings indicated that GLI2 contributes to the growth of cervical cancer in vivo.

We then determined whether GLI3 was capable of effecting tumor growth in vivo. Transfected Caski cells (GLI3-shR1-Caski and Ctl-shR2-Caski cells) were injected subcutaneously into the right flank of nude mice. Consistent with the data obtained from in vitro studies, depletion of GLI3 showed no effect on tumor growth in Caski xenograft tumors (Fig. 7d, e) and on the number of proliferative tumor cells stained with Ki-67 (Fig. 7f). We also confirmed that the tumors formed by GLI3-shR1-Caski cells had sustained GLI3 knockdown as reflected by significantly reduced GLI3 mRNA measured with qRT-PCR (Fig. 7g), confirming that the similar tumorigenic property between the control and GLI3-shR cells was not due to the loss of GLI3 knockdown. Taken together, these findings further support the notion that GLI3 is not a tumor promoter in cervical carcinogenesis.

\section{High expression of GLI2, but not GLI3, is associated with an unfavorable prognosis in patients with cervical cancer}

We then investigated the association between the expression of GLI $/ 3$ and clinical outcome of cervical cancer in TCGA dataset. High expression of GLI2 was found to be related to a poor overall survival of cervical cancer, whereas GLI3 showed no effect on overall survival of cervical cancer (Fig. 8a, b).

\section{Discussion}

The genes involved in the Hh signaling pathway have been shown to be upregulated in cervical carcinoma [9] and treatment with the Hh pathway inhibitor, KAADcyclopamine, induced a significant inhibition of cell proliferation in four cervical cancer cell lines [16]. GLI2, a 

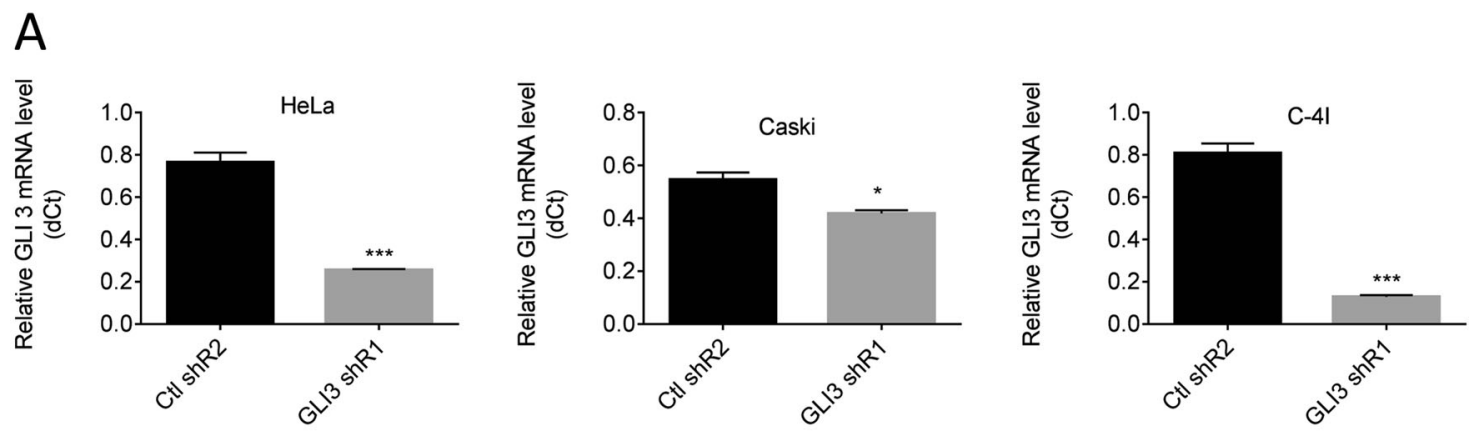

B

HeLa

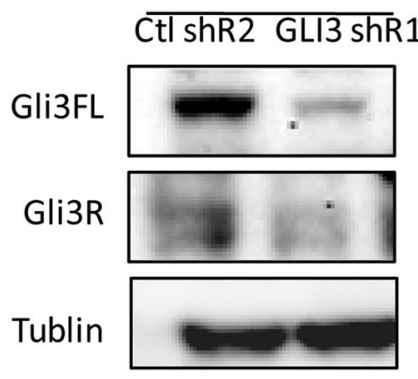

Caski

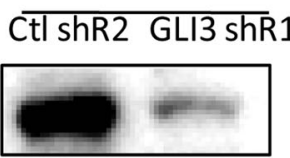

C-4 I
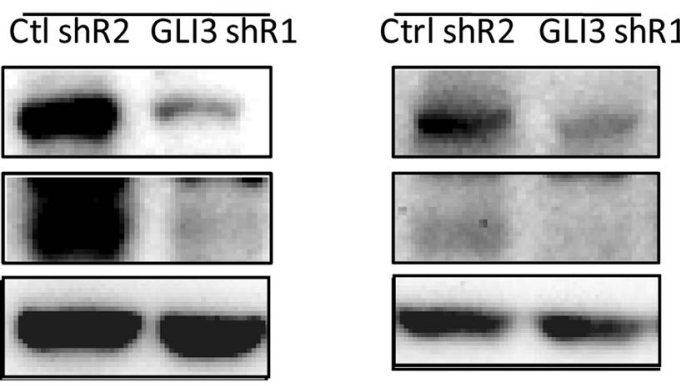

C

HeLa
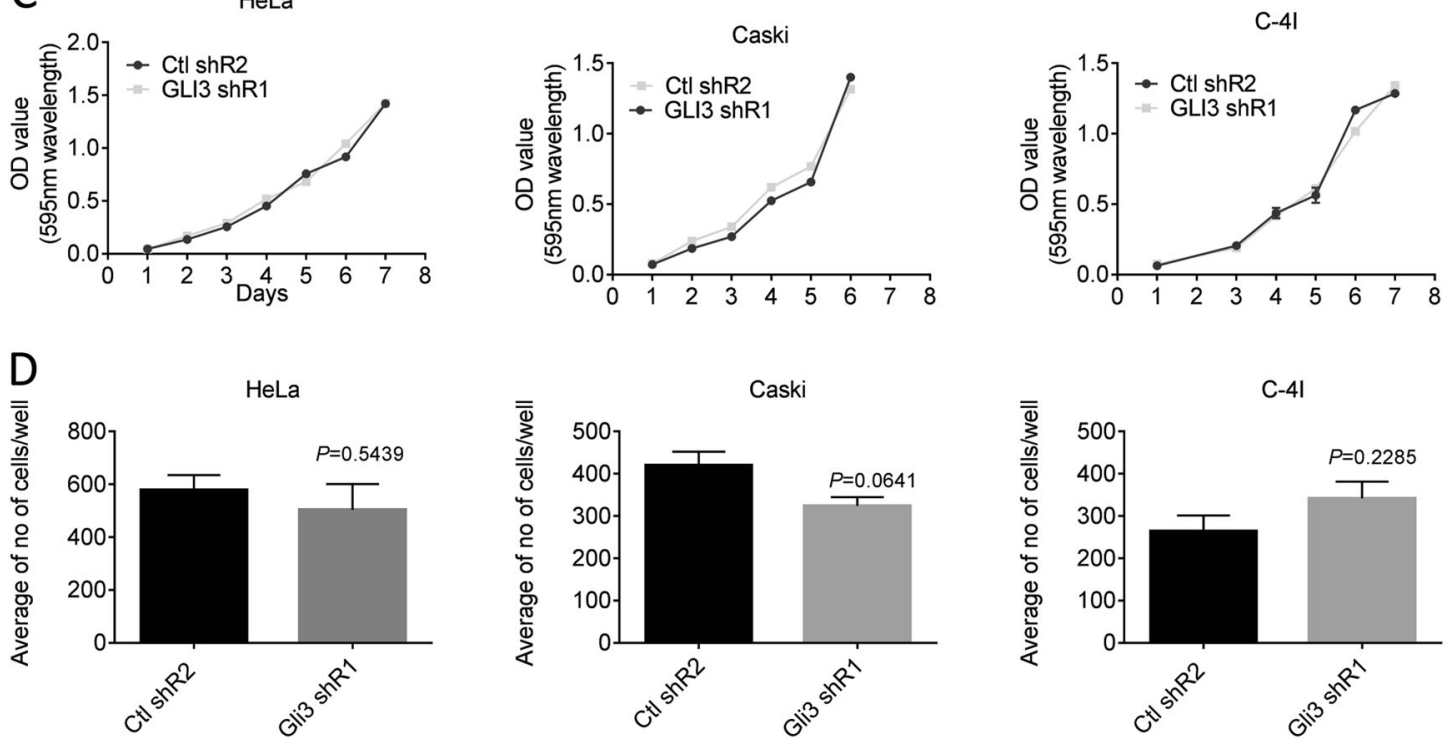

Fig. 6 Stable knockdown of GLI3 had no effect on proliferation and migration in vitro. GLI3 shRNA or matched control shRNA vector were transfected into HeLa, Caski and C-4I cells via lentiviral infection. (a) Confirmation of GLI3 stable knockdown in transcript level by qRT-PCR and (b) in protein level by western blotting. Tublin level was used to validate equal sample loading for the western blotting and

GAPDH transcript was used for normalization of the qRT-PCR assay. Both the full length (GLI3FL) and repressor (GLI3R) versions of GLI3 protein were detected. c GLI3 knockdown had no effect on cell growth by MTT assay. d GLI3 knockdown had no effect on migration in cervical cancer cells. Data presented were mean \pm SEM. $* P<0.05$; *** $P<0.001$ with two-tailed Student's $t$-test

primary mediator of $\mathrm{Hh}$ signaling, was reported to be over-expressed in cervical cancer compared with normal cervical tissues. Indeed, our present data also showed that GLI2 mRNA and protein are readily detectable in both cervical cancer cell lines and cervical cancer tissues. However, the function of GLI2 in the cervical carcinogenesis and whether lowering the expression of
GLI2 can suppress tumor growth have not been reported. Our current results, for the first time, showed that depletion of GLI2 could suppress the proliferation and migration of cervical cancer cells in vitro and their tumorigenicity in vivo. These data were in line with previous observations in other cancer models, where the inhibition of GLI2 was efficient in suppressing tumorigenic properties of prostate 
A

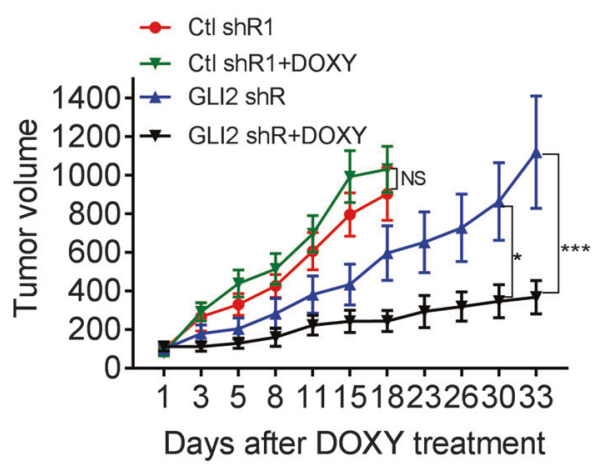

B

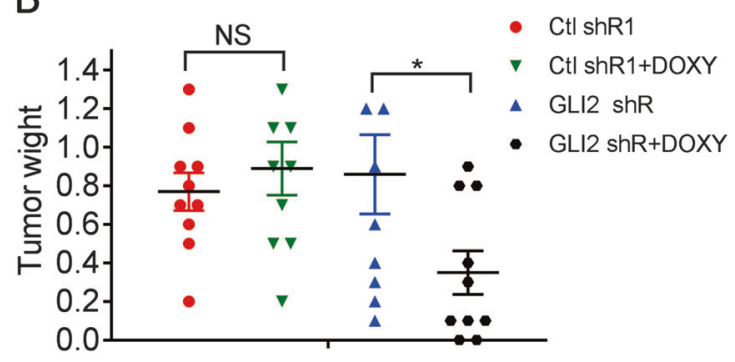

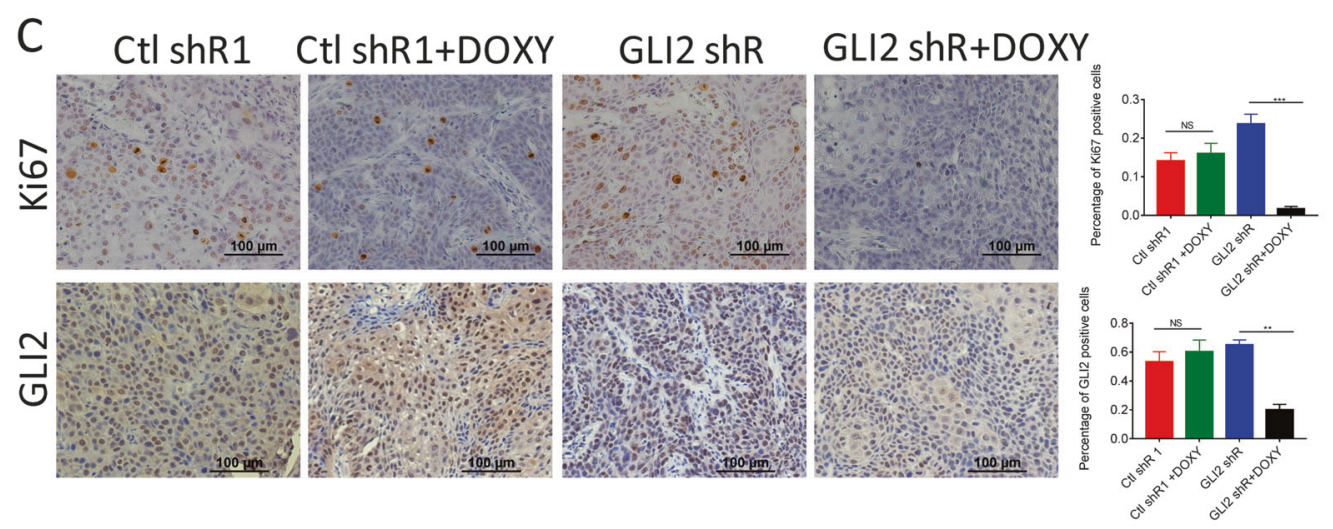

D

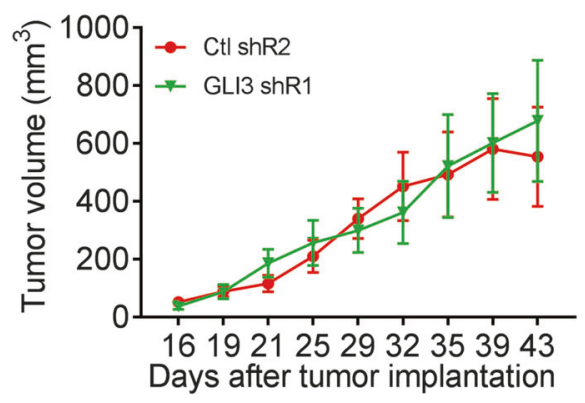

$\mathrm{F}$

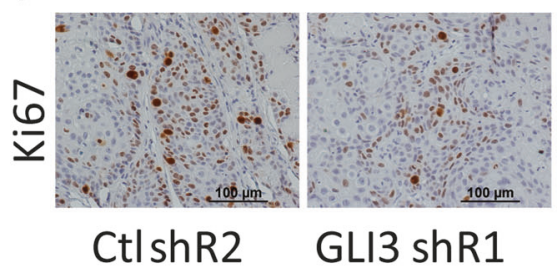

$\mathrm{E}$

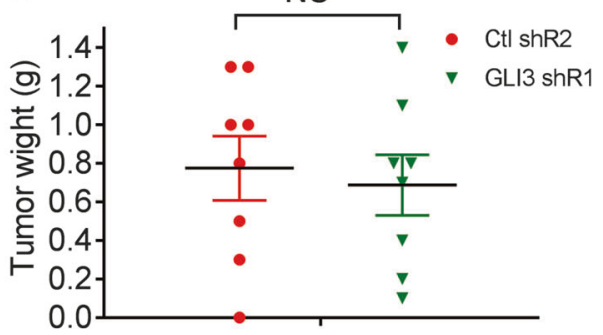

G

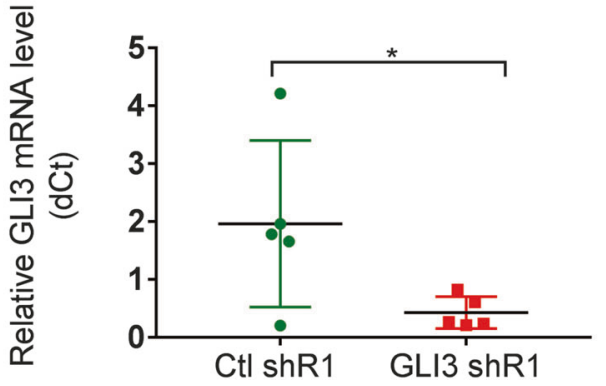

cancer cells [17] and hepatoma cells both in vitro and in vivo [18, 19].

Mounting evidence implicates GLI2 in cell-cycle progression at the restriction point. Nagao et al. reported that silencing GLI2 prevented human osteosarcoma growth by inducing a G1 cell-cycle arrest via p21 (cip1) upregulation with a subsequent inhibition of cyclin D1, SKP2, and phosphorylated $\mathrm{Rb}$ [20]. Zhang et al. reported that knockdown of GLI2 resulted in G1 arrest with concomitant reduction of cyclin D1 and cyclin E2 and up-regulation of p21 (WAF1) levels in human hepatocellular carcinoma cells [19]. Similar effects involving a G0/G1 arrest caused by silencing of GLI2 have been reported in human vascular smooth muscle cells [21] and myofibroblasts [22]. Clearly, 
Fig. 7 Knockdown of GLI2, but not GLI3, inhibited tumor growth in vivo. a Growth curve of tumors formed by GLI2 shRNA and matched Ctl shRNA Caski cells in female nude mice treated with or without doxycycline in drinking water. The tumor volume was calculated using the formula: $v=$ length $\times$ width $\times$ width $\times 0.5$. Each data point represented the mean \pm SEM of ten tumors. $\mathbf{b}$ Tumors excised from euthanized mice were weighed at the end of experiment. Each dot represented a tumor. Mean \pm SEM was also presented. $\mathbf{c}$ IHC staining of Ki-67 and GLI2 in tumor sections from the four groups of experimental mice. Representative IHC pictures were presented for each staining from tissue sections of three tumors in each group. Scale bar, $100 \mu \mathrm{m}$. Percent of Ki-67 or GLI2-positive cells were plotted. **P $<0.01$; *** $P<0.001$; with one-way ANOVA and Tukey-Kramer post hoc test. d Tumor volumes of GLI3 shRNA and matched Ctl shRNA Caski cell-formed tumors were calculated using the formula: $v=$ length $\times$ width $\times$ width $\times 0.5$. Each data bar represented the mean \pm SEM of ten tumors. e Individual tumor weights from the two group mice at the end of experiment along with the mean \pm SEM were presented. f Representative IHC staining for Ki-67 in xenograft tumor sections from the two experimental groups were presented. Scale bar, $100 \mu \mathrm{m}$. g qRT-PCR analysis for GLI3 in five GLI3 shRNA and matched Ctl shRNA Caski xenograft tumors were plotted. $* P<0.05$; with two-tailed Student's $t$-test

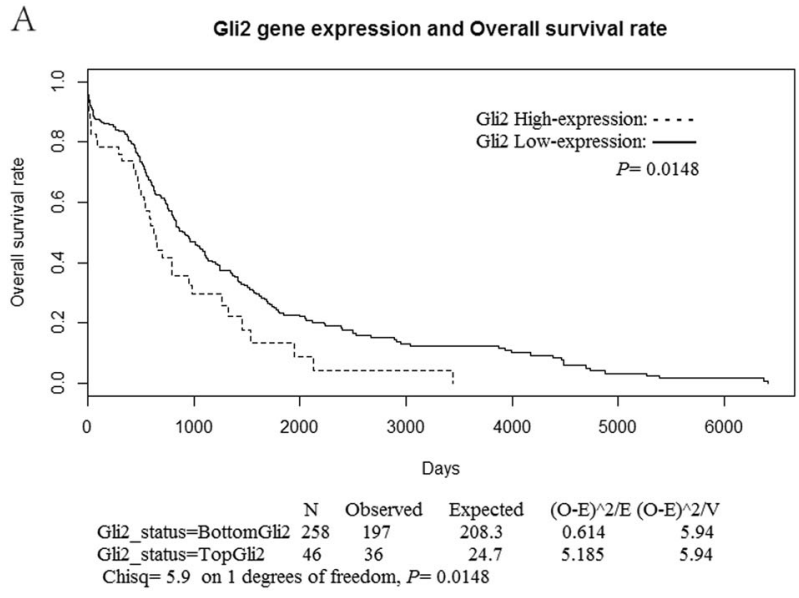

B

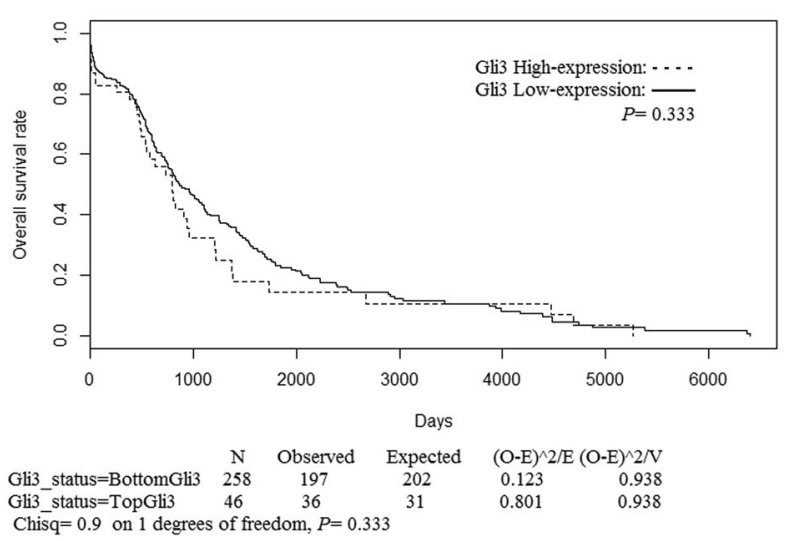

Fig. 8 Kaplan-Meier analysis of GLI2 and GLI3 mRNA expression and overall survival of 304 cervical cancer patients from TCGA datasets. High expression of GLI2 was related to a poor overall survival of cervical cancer (a), whereas GLI3 showed no association with overall survival of cervical cancer (b) our current results were consistent with all of these findings that knockdown GLI2 resulted in cell-cycle arrest at G0/G1, with reduction of cyclin D1 expression and upregulation of p21 and p27 levels, whereas overexpression of GLI2 increased proliferation. Intriguingly, while previous studies reported that GLI2 gene silencing sensitized human hepatoma cells [18, 19] and basal cell carcinoma [23] to tumor necrosis factor-related apoptosis by downregulating c-FLIP and Bcl-2, our result showed that GLI2 knockdown showed no effect on apoptosis using two apoptosis assays, including ELISA assay and Annexin-V FITC staining. Future studies are needed to determine whether GLI2 are necessary for preventing apoptosis of cervical cancer cells when exposing to apoptosis-inducing agents.

The AKT pathway plays a pivotal role in the etiology and progression of cancer by affecting cell metabolism, growth, survival, proliferation, migration, and angiogenesis $[24,25]$. Aberrant over-expression of p-AKT is a common feature in both early and advanced cervical cancers [12-15]. $\mathrm{Du}$ et al. reported that the positive staining frequency of $\mathrm{p}$ AKT in chronic cervicitis tissues, cervical intraepithelial neoplasia, and cervical carcinoma tissues was $21.05 \%$, $66.67 \%$, and $92.31 \%$, respectively, suggesting p-AKT expression increased as tumor state progressed from lowgrade cervical intraepithelial neoplasia to high-grade cervical intraepithelial neoplasia to cancer [12]. Furthermore, high p-AKT expression was a risk factor for disease-free survival and overall survival of cervical cancer [13]. Consistent with the previous data, our results showed that ectopic expression of AKT with an increased p-AKT level promoted cervical cancer proliferation. Furthermore, AKT expression rescued the cells' growth arrest caused by lowing GLI2 expression confirming that AKT signaling was a downstream target of GLI2 mediating its growth-promoting activity.

Because both GLI2 and GLI3 are over-expressed in cervical cancer and can act as repressors or activators, we initially hypothesized that knockdown of either GLI2 or GLI3 might have the same effect on cervical cancer growth. While GLI2 knockdown inhibited cervical cancer cell proliferation and migration in vitro and xenograft growth in vivo, GLI3 knockdown did not affect cervical cancer cell proliferation and migration. In vivo study further demonstrated that lowering the expression of GLI3 did not suppress cervical cancer xenograft growth. This observation is in contrast to the only previous report from Wen et al. [26]. A probable explanation could be due to off-target effects of various sequences of the GLI3 siRNA and shRNAs used in the two studies. On the other hand, the clinical data from TCGA dataset showed that the expressions of GLI2, but not GLI3, in the cervical cancer tissues was associated with a worse overall survival by Kaplan-Meier analysis, which supported the notion that GLI2, but not GLI3, functioned as 
a tumor-promoter in cervical cancer. Taken together, our results directly implicated GLI2, but not GLI3, as a potential therapeutic target in cervical cancer.

Acknowledgements This study was in part supported by grants from NIH R01CA172886 and R01CA196214 to L. -Z. S., and from Key Lab of Wenzhou city-Gynecological Oncology to X. Q. Z. Additional support was provided by the Flow Cytometry Shared Resource of the Cancer Therapy and Research Center, which is supported by the NIH NCI Cancer Center Support Grant P30 CA054174-17. H. -Y. Z. and Qi Shen were supported by a fellowship from the Second Affiliated Hospital of Wenzhou Medical University and Key Lab of Wenzhou city-Gynecological Oncology. L. X. and B. W. were in part supported by a fellowship from Xiangya School of Medicine, Central South University, Hunan, China. X. G. was supported by the CPRIT Research Training Award RP140105 and RP170345.

\section{Compliance with ethical standards}

Ethics statement All animal experiments were conducted following appropriate guidelines. They were approved by the Institutional Animal Care and Use Committee and monitored by the Department of Laboratory Animal Resources at the University of Texas Health Science Center at San Antonio.

Conflict of interest The authors declare that they have no conflict of interest.

\section{References}

1. Torre LA, Bray F, Siegel RL, Ferlay J, Lortet-Tieulent J, Jemal A. Global cancer statistics, 2012. CA Cancer J Clin. 2015;65:87-108.

2. Jiang J, Hui CC. Hedgehog signaling in development and cancer. Dev Cell. 2008;15:801-12.

3. Robbins DJ, Fei DL, Riobo NA. The Hedgehog signal transduction network. Sci Signal. 2012;5:re6.

4. Briscoe J, Therond PP. The mechanisms of Hedgehog signalling and its roles in development and disease. Nat Rev Mol Cell Biol. 2013;14:416-29.

5. Humke EW, Dorn KV, Milenkovic L, Scott MP, Rohatgi R. The output of Hedgehog signaling is controlled by the dynamic association between Suppressor of Fused and the Gli proteins. Genes Dev. 2010;24:670-82.

6. Hui CC, Angers S. Gli proteins in development and disease. Annu Rev Cell Dev Biol. 2011;27:513-37.

7. Chaudary N, Pintilie M, Hedley D, et al. Hedgehog pathway signaling in cervical carcinoma and outcome after chemoradiation. Cancer. 2012;118:3105-15.

8. Chen H, Wang J, Yang H, Chen D, Li P. Association between FOXM1 and hedgehog signaling pathway in human cervical carcinoma by tissue microarray analysis. Oncol Lett. 2016;12:2664-73.

9. Xuan YH, Jung HS, Choi YL, et al. Enhanced expression of hedgehog signaling molecules in squamous cell carcinoma of uterine cervix and its precursor lesions. Mod Pathol. 2006;19: 1139-47.
10. Vishnoi K, Mahata S, Tyagi A, et al. Cross-talk between human Papillomavirus oncoproteins and hedgehog signaling synergistically promotes stemness in cervical cancer cells. Sci Rep. 2016;6:34377.

11. Roessler E, Ermilov AN, Grange DK, et al. A previously unidentified amino-terminal domain regulates transcriptional activity of wild-type and disease-associated human GLI2. Hum Mol Genet. 2005;14:2181-8.

12. Du CX, Wang Y. Expression of P-Akt, NFkappaB and their correlation with human papillomavirus infection in cervical carcinoma. Eur J Gynaecol Oncol. 2012;33:274-7.

13. Cho H, Noh KH, Chung JY, et al. Synaptonemal complex protein 3 is a prognostic marker in cervical cancer. PLoS ONE. 2014;9: e98712.

14. Choi SK, Hong YO, Lee WM, et al. Overexpression of PI3Kp110alpha in the progression of uterine cervical neoplasia and its correlation with pAkt and DJ-1. Eur J Gynaecol Oncol. 2015;36:389-93.

15. Bumrungthai $\mathrm{S}$, Munjal K, Nandekar S, et al. Epidermal growth factor receptor pathway mutation and expression profiles in cervical squamous cell carcinoma: therapeutic implications. J Transl Med. 2015;13:244.

16. Chan DW, Liu VW, Leung LY, et al. Zic2 synergistically enhances Hedgehog signalling through nuclear retention of Gli1 in cervical cancer cells. J Pathol. 2011;225:525-34.

17. Thiyagarajan S, Bhatia N, Reagan-Shaw S, et al. Role of GLI2 transcription factor in growth and tumorigenicity of prostate cells. Cancer Res. 2007;67:10642-6.

18. Zhang DW, Li HY, Lau WY, et al. Gli2 silencing enhances TRAIL-induced apoptosis and reduces tumor growth in human hepatoma cells in vivo. Cancer Biol Ther. 2014;15: 1667-76.

19. Zhang D, Liu J, Wang Y, Chen J, Chen T. shRNA-mediated silencing of Gli2 gene inhibits proliferation and sensitizes human hepatocellular carcinoma cells towards TRAIL-induced apoptosis. J Cell Biochem. 2011;112:3140-50.

20. Nagao H, Ijiri K, Hirotsu M, et al. Role of GLI2 in the growth of human osteosarcoma. J Pathol. 2011;224:169-79.

21. Li F, Duman-Scheel M, Yang D, et al. Sonic hedgehog signaling induces vascular smooth muscle cell proliferation via induction of the G1 cyclin-retinoblastoma axis. Arterioscler Thromb Vasc Biol. 2010;30:1787-94.

22. Kramann R, Fleig SV, Schneider RK, et al. Pharmacological GLI2 inhibition prevents myofibroblast cell-cycle progression and reduces kidney fibrosis. J Clin Invest. 2015;125:2935-51.

23. Kump E, Ji J, Wernli M, Hausermann P, Erb P. Gli2 upregulates cFlip and renders basal cell carcinoma cells resistant to death ligand-mediated apoptosis. Oncogene. 2008;27:3856-64.

24. Fresno Vara JA, Casado E, de Castro J, Cejas P, Belda-Iniesta C, Gonzalez-Baron M. PI3K/Akt signalling pathway and cancer. Cancer Treat Rev. 2004;30:193-204.

25. Mundi PS, Sachdev J, McCourt C, Kalinsky K. AKT in cancer: new molecular insights and advances in drug development. Br J Clin Pharmacol. 2016;82:943-56.

26. Wen SY, Lin Y, Yu YQ, et al. miR-506 acts as a tumor suppressor by directly targeting the hedgehog pathway transcription factor Gli3 in human cervical cancer. Oncogene. 2015;34:717-25. 